\title{
DISEÑO DE UNA ESCALA PARA LA EVALUACIÓN DEL COMPORTAMIENTO CREATIVO EN DIFERENTES DOMINIOS
}

\section{A SCALE FOR THE EVALUATION OF CREATIVITY BEHAVIOR IN DIFFERENT DOMAINS: DEVELOPMENT AND DESIGN}

\author{
María Aranguren \\ Pontificia Universidad Católica Argentina, Argentina \\ Consejo Nacional de Investigaciones Científicas y Tecnológicas, Argentina \\ Natalia Irrazabal \\ Centro de Investigaciones en Psicología y Psicopedagogía - U.C.A.-, Argentina \\ Consejo Nacional de Investigaciones Científicas y Tecnológicas, Argentina
}

\begin{abstract}
Resumen: Este estudio se centró en diseñar y analizar -preliminarmente- las propiedades psicométricas de una escala de evaluación de la creatividad en diferentes áreas: literatura; música; expresión corporal (danza/ teatro); diseño/artesanías; artes plásticas; empresas/negocios; arquitectura/construcción, ciencia/tecnología; y creatividad cotidiana. Los análisis factoriales exploratorios $(n=258)$ generaron 4 factores: F1 Artes y diseño; F2 Literatura y música; F3 Expresión corporal y F4 Empresas y negocios. Los resultados indican una buena consistencia interna de la ECC y de cada subescalas y proveen evidencias sobre validez convergente con constructos teóricos como, personalidad creativa, autoeficacia creativa y autoeficacia general. Dichos resultados indican que, tanto la ECC como sus subescalas muestran correlación baja, aunque estadísticamente significativa, con Escala de Personalidad Creativa (Gough, 1979), Escala de Autoeficacia Creativa (Yi, Scheithauer, Lin \& Schwarzer, 2008) y Escala de Autoeficacia General (Jerusalem \& Schwarzer, 1992). Se aportan evidencias de validez criterial, por grupos contrastados, observándose diferencias estadísticamente significativas en puntuaciones promedios del total de la ECC y en subescalas de Artes/ diseño, Literatura/ música y Expresión corporal, entre participantes que desarrollaron alguna actividad artística versus aquellos que no. Se informan diferencias según sexo, edad y nivel educativo para totales de la ECC y cada subescala.
\end{abstract}

Palabras clave: Evaluación, creatividad, diseño de escala, confiabilidad, validez.

\begin{abstract}
This study focused on the design and preliminar analysis of the psychometric properties of a creativity assessment scale in different areas: literature, music, body language (dance/theater), design/crafts, fine arts, business, architecture/construction, science/technology, and everyday creativity. The exploratory factor analysis $(\mathrm{n}=258)$ rendered 4 factors: F1 Art and Design; F2 literature and music, F3 body language and F4 business. These results indicate good internal consistency of the scale and sub-scales, and also provide evidence of the convergent validity of these with other theoretical constructs such as creative personality, creative self-efficacy and overall self-efficacy. Both the ECC and its subscales showed low correlation, although statistically significant, to the Creative Personality Scale (Gough, 1979), the Creative Self-Efficacy Scale (Yi, Scheithauer, Lin \& Schwarzer, 2008) and the Overall Self-Efficacy Scale (Jerusalem \& Schwarzer, 1992). Evidence ofcriteria validity for contrasted groups is provided. Statistically significant differences for the overall average scores and the fine arts/design, literature/music and body language subscales when those individuals who pursue an artistic activity are compared whith those who do not. Sex, age and education background differences for the overall scale and the different subscales are reported.
\end{abstract}

Keywords: Assessment, creativity, scale design, reliability, validity.

\section{INTRODUCCIÓN}

El estudio de la creatividad ha recibido un fuerte desarrollo desde que en el año 1950, Guilford impulsara a los psicólogos y profesionales afines, a la investigación científica de este atributo. Sin embargo, desde ese entonces se han dado algunos obstáculos en el estudio académico o científico de la creatividad, entre los que se destacan: a) la dificultad referida a la definición de la creatividad y los criterios utilizados para evaluarla; b) el

Correspondencia: Prof. María Aranguren Facultad de Psicología Pontificia Universidad Católica Argentina.

Correo Electrónico: maria_aranguren@uca.edu.ar 
hecho de que algunos estudios consideren la creatividad como el resultado excepcional de la conjunción de estructuras o procesos ordinarios, focalizándose en el estudio de dichas estructuras y desmereciendo el estudio de la creatividad como un proceso singular; c) la común asociación entre la creatividad y aspectos místicos-espirituales en el imaginario popular y; d) la impresión de que el estudio de la creatividad carece de teorías subyacentes que fundamenten los resultados encontrados -a menudo generada por abordajes poco serios, comerciales o extremadamente pragmáticos(Sternberg \& Lubart, 1999).

Entre los aspectos mencionados, uno de los más relevantes y más controvertido es el referido a la definición de la creatividad y su evaluación. Comúnmente, se suele definir a la creatividad como facultad de crear, capacidad para producir o introducir algo por primera vez (Real Academia Española, 2001). También, se la asocia con conceptos como el juego, la imaginación, el genio, la fantasía y la habilidad para innovar. A pesar de ello, no se ha de confundir el concepto que la mayoría de las personas comparten acerca de lo que es la creatividad con la necesidad de elaborar y acordar una definición precisa en el ámbito académico y científico. Así, diferentes autores afirman que hasta que no se logren aunar las características y atributos principales de lo que se considera que es la creatividad en el ámbito académico, el desarrollo de este campo deberá seguir afrontando desarrollos dispares, resultados contradictorios y discrepancias en los criterios de evaluación de la misma (Donolo \& Elisondo, 2007; Plucker, Beghetto, \& Dow, 2004).

Otra de las dificultades en el estudio de la creatividad, es que la misma puede ser entendida como un atributo general de la persona o como un atributo específico referido al dominio en el que se den los logros creativos. Así algunos autores sostienen que la creatividad es un atributo personal e independiente del dominio (Plucker, 1998) mientras que otros autores postulan que para estudiarla se ha de tener en consideración el contenido o dominio en el que se desarrolle la misma (Baer, 1998). En consonancia con esta última idea, diversos estudios señalan que para ser creativo es necesario tiempo, experiencia y cierta experticia en un área particular del saber (Baer, \& Kaufman, 2005; Hayes, 1981; Weisberg, 2006). La creatividad se define entonces, no sólo de acuerdo a ciertas características de personalidad o teniendo en cuenta únicamente el rendimiento en determinados procesos cognitivos, sino también incluyendo los logros adquiridos por las personas en determinadas áreas en función de su formación y experticia.

Actualmente, se distinguen diferentes enfoques para el estudio de la creatividad: el enfoque psicodinámico; el psicométrico; el cognitivo-experimental; el social y sistémico, en el que se incluyen variables de personalidad, motivacionales y socio-culturales; el enfoque historiométrico y los estudios de casos y biográficos (Plucker \& Renzulli, 1999; Sternberg \& Lubart, 1999). Dentro del enfoque psicométrico, se destacan cuatro áreas específicas dirigidas a la evaluación del proceso creativo, la personalidad creativa, los productos creativos y las características contextuales que influyen en el desarrollo de la creatividad. En este sentido, desde 1950 hasta hoy, han sido diseñados diferentes instrumentos dirigidos a evaluar cada uno de estos aspectos. También se encuentran disponibles algunas escalas e inventarios que tienen por finalidad indagar acerca de diversos comportamientos, actividades y logros creativos realizados por las personas en su vida cotidiana. En este grupo se encuentran el Inventario de Comportamiento Creativo (Creativity Behavior Inventory) de Hocevar (1979; 1980); la Escala de Creatividad a lo largo de la vida (Lifetime Creativity Scales; Richards, Kinney, Benet, \& Merzel, 1988) y el Cuestionario de Logros Creativos (Creative Achievement Questionnaire; Carson, Peterson, \& Higgings, 2005).

En Argentina, se encuentran muy pocas investigaciones empíricas en el ámbito de la psicología de la creatividad (e.g. Armesto, 2001; Donolo \& Rinaudo, 2008; Krumm, 2004), y los instrumentos disponibles para su evaluación son escasos. Entre estos, se puede nombrar la adaptación argentina de la Escala de Personalidad Creativa de Garagordobil (2004) en su versión de heteroevaluación, (Krumm y Lemos, 2011). También se encuentran algunos estudios premiliminares acerca de las propiedades psicométricas del Test de Pensamiento Creativo de Torrance verbal 
(Torrance Test of Creative Thinking verbal; Torrance, 1974) realizados por Krumm y Lemos (2010). Teniendo en consideración la escasez de instrumentos para el estudio de la creatividad, el presente estudio tuvo por objeto diseñar un instrumento para la evaluación del comportamiento creativo. De este modo, a través del diseño de la Escala de Comportamiento Creativo (ECC) se intenta realizar un aporte a un área en desarrollo como es la investigación de los rasgos y procesos creativos de las personas.

\section{EVALUACIÓN DEL COMPORTAMIENTO CREATIVO}

Una de las definiciones que ha obtenido mayor aceptación por parte de los académicos que trabajan en el área, y desde la cual parte este estudio, postula que la creatividad es una forma de pensar cuyo resultado son productos que poseen novedad y valor (Romo, 1997). Del mismo modo, para Barron (1955) un producto creativo debe reunir dos características: (a) debe ser original y; (b) debe responder o estar consonancia con las necesidades de la realidad. En otras palabras, cualquier producto creativo debe ser novedoso y útil, ya se trate de un poema, una composición, un descubrimiento científico o una obra de construcción. Siguiendo estas ideas en el presente trabajo se define el comportamiento creativo como todo comportamiento que tenga por finalidad generar un producto, una idea o una obra que tenga cierto grado de novedad y valor.

Habitualmente se suele distinguir entre dos niveles o grados de creatividad: la creatividad diaria (everyday creativity) y la creatividad sobresaliente (eminent creativity). La creatividad diaria está referida a las contribuciones originales que realizan las personas en su vida cotidiana, aportando un nuevo significado a las situaciones y/u objetos con los que interactúan, y promoviendo de esta forma el crecimiento personal (Richards, 2007). Desde esta perspectiva, todas las personas pueden ser más o menos creativas, esto es, la creatividad es un atributo que puede darse en distintos grados. Concretamente, la creatividad diaria puede verse en la resolución de los problemas cotidianos - laborales, personales, del hogar -, en la improvisación de una receta de cocina cuando no se cuenta con los ingredientes necesarios, en la decoración de la casa, el cuidado del jardín o la ideación de un nuevo juego para entretener a los chicos (Runco, 2003).

Otros autores han preferido distinguir entre lo que se ha dado en llamar la mini-c, little-c y la Big-C. Las conceptualizaciones referidas a la mini-c y little-c son, en general, muy similares a lo que se ha denominado como creatividad diaria mientras que la creatividad sobresaliente se correspondería con la Big-C. El grado inferior de creatividad llamado mini $c$ se define como "una interpretación novedosa y personalmente significativa de las experiencias, las acciones y los eventos" (Beghetto \& Kaufman, 2007, p. 73). Los niveles de creatividad llamados little-c y Big$C$, difieren en cuanto al nivel de logros creativos alcanzados, pero ambos implican un producto o logro que tenga cierto grado de novedad y de valor y un juicio externo que garantice dichas características; en tanto que cuando se habla de mini-c, la novedad y el significado del logro alcanzado es dado por un juicio intrapersonal.

El constructo de mini-c fue propuesto con tres objetivos principales: (a) subrayar la relación entre el aprendizaje y la creatividad; (b) poder evaluar la génesis del comportamiento creativo; (c) Ilenar un vacío existente en la conceptualización actual de los niveles de la creatividad permitiendo la evaluación del esfuerzo y el potencial creativo de las personas a pesar de que las mismas no hayan sido congraciadas con ningún mérito aún. En la Tabla 1 (páguina siguiente) se muestran las diferencias entre los distintos niveles de creatividad.

Estas conceptualizaciones se encuentran relacionadas con los aportes de los psicólogos humanistas, tales como Rogers (1970) y Maslow (1968), quienes sostienen que la creatividad es una característica presente en todas personas, característica que hace a su autorrealización así como a la actualización de sus potencialidades. A su vez, las mismas han ido adquiriendo mayor interés en las investigaciones actuales de la creatividad. A pesar de que el estudio de la creatividad sobresaliente sea relevante porque permite conocer las características de la creatividad en su más alta expresión, estos estudios no abarcan el espectro completo de lo que es la creatividad ni de sus manifestaciones. 
Tabla 1.

Distinción entre mini-c, little-c y Big-C.

\begin{tabular}{|c|c|c|c|}
\hline & Big-C & Little-c & Mini-c \\
\hline Alcance & $\begin{array}{l}\text { Logros creativos que } \\
\text { cambian un campo } \\
\text { disciplinar. }\end{array}$ & $\begin{array}{l}\text { Logros creativos que pueden llegar } \\
\text { a representar una contribución } \\
\text { sólida a un campo disciplinar. }\end{array}$ & $\begin{array}{l}\text { Creatividad } \\
\text { intrapersonal que } \\
\text { forma parte del proceso } \\
\text { de aprendizaje. }\end{array}$ \\
\hline $\begin{array}{l}\text { Ejemplo de } \\
\text { un producto }\end{array}$ & $\begin{array}{l}\text { Una pintura de Van } \\
\text { Gogh }\end{array}$ & $\begin{array}{l}\text { Una pintura realizada para un } \\
\text { amigo querido. }\end{array}$ & $\begin{array}{l}\text { Un boceto de un } \\
\text { estudiante con varias } \\
\text { combinaciones de luz y } \\
\text { sombra. }\end{array}$ \\
\hline $\begin{array}{l}\text { Ejemplo de } \\
\text { una persona }\end{array}$ & Bill Gates & Un colega. & $\begin{array}{l}\text { Un estudiante de artes } \\
\text { avanzado. }\end{array}$ \\
\hline Evaluación & $\begin{array}{l}\text { Enfoques } \\
\text { historiométricos. }\end{array}$ & Test psicométricos & $\begin{array}{l}\text { Métodos } \\
\text { microgenéticos. }\end{array}$ \\
\hline Experiencia & Más de 10 años & $\begin{array}{l}\text { Algún grado de escolarización o } \\
\text { experiencia alcanzado. }\end{array}$ & Ninguno. \\
\hline
\end{tabular}

Fuente: Beghetto y Kaufman, 2007, p. 76.

Tal como señalan Beghetto y Plucker (2007), la creatividad diaria no supone grandes revoluciones científicas o artísticas pero sí implica algún grado de novedad y de valor en los comportamientos, acciones y actividades que realizan las personas en el día a día.

Al diseñar un instrumento destinado a evaluar la creatividad uno debe distinguir no sólo a que faceta de la creatividad se encuentra referido -persona creativa, proceso creativo, producto creativo o al ambiente-, sino además distinguir entre los distintos grados de creatividad a los que se dirige. En consonancia con las ideas mencionadas, la ECC tiene por objeto estudiar el comportamiento creativo de las personas en distintos dominios, apuntando a evaluar no la creatividad sobresaliente, sino la creatividad diaria, integrando los niveles de little-c y mini-c.

\section{ANTECEDENTES DE INSTRUMENTOS REFERIDOS AL COMPORTAMIENTO CREATIVO}

En la literatura se encuentran distintos modos de evaluar el comportamiento creativo. En primer lugar, en algunos estudios se les solicita a un grupo de expertos valorar la creatividad sobresaliente. Por ejemplo, MacKinnon (1962) pide a un número determinado de profesores de arquitectura identificar a los arquitectos contemporáneos más influyentes en el área. En segundo lugar, ciertas investigaciones combinan la utilización de juicios de no expertos y el uso de instrumentos psicométricos. En este grupo se encuentran las investigaciones realizadas por Ludwig (1992) quien desarrolló la Escala de Logros Creativos (Creative Achievement Scale - CAS), en la que se les pide a jueces no expertos, previamente entrenados en el uso de la escala, que evalúen los logros o comportamientos creativos de personas fallecidas de distintas profesiones a través de las biografías publicadas.

En tercer lugar, varias investigaciones en donde aplican exclusivamente pruebas psicométricas. Tal es el caso de los estudios realizados por Richards et al. (1988) quienes diseñaron las Escalas de Creatividad a lo largo de la vida (Lifetime Creativity Scale). Dichas escalas fueron incluidas end distintas entrevistas para poder relevar información acerca de la cantidad y cualidad de los productos y comportamientos creativos realizados por los participantes a lo largo de su vida. En este grupo también se pueden mencionar algunos inventarios de comportamientos, logros o productos creativos como los desarrollados por Holland y Nichols (1964), Torrance (1972), Hocevar (1979), o Carson et al. (2005). Algunos autores han sugerido que los inventarios autoadmistrables son uno de los métodos más confiables para evaluar tanto los logros como el talento creativo (Hocevar, 1981; Hocevar \& Bacherlor, 1989). En este sentido, una de las bondades de estos inventarios es su validez predictiva. Los logros obtenidos, las actividades realizadas así como también el comportamiento pasado es, en general, 
el mejor predictor del comportamiento futuro (Hocevar, 1981).

La mayoría de estos inventarios consiste en una lista de actividades, comportamientos y logros creativos, en los que se les pide a los participantes señalar aquellos que han realizado a lo largo de un determinado período de tiempo o a lo largo de su vida. En general, todos estos inventarios evalúan en la creatividad en distintas áreas: música, artes, ciencia, literatura, arquitectura, teatro, entre otros. Esta característica se fundamenta en que para muchos autores la creatividad debe ser estudiada en relación al dominio en el que tenga lugar, dado que una persona puede ser creativa en un área pero no en otras ya sea porque en otras áreas no muestra la misma motivación, interés o dedicación (Hocevar, 1981; Ward, Smith, \& Finke, 1999). En consonancia con estas ideas, tanto el modelo componencial de Amabile (1983) como el modelo sistémico de Csikszentimihalyi (1998), señalan la importancia que tiene el campo en el desarrollo de la creatividad. Desde el modelo componencial de Amabile (1983) se destacan las destrezas relevantes para el campo conocimiento sobre el campo, destrezas técnicas requeridas y talento especial relevante para el campo- y la motivación por la tarea actitudes hacia la tarea y percepciones acerca de la propia motivación para realizar la tarea-, como dos aspectos centrales de la creatividad. Para Csikszentimihalyi (1998), la creatividad no es un proceso individual sino un proceso que se da en interacción entre tres sistemas: la persona, el campo y el ámbito. Por otro lado, también los hallazgos de Hayes (1981) señalan que para que una persona pueda realizar un logro creativo en algún área, debe haberle dedicado al menos 10 años de trabajo y esfuerzo. Todos estos aportes llevan a pensar que de querer evaluar la creatividad uno debe contemplar el área en el que tenga lugar.

\section{CONSIDERACIONES SOBRE LA ESCALA DE COMPORTAMIENTO CREATIVO}

El presente estudio tuvo por objetivo diseñar una escala destinada a la evaluación del comportamiento creativo en diferentes áreas. Para tal fin, se realizó una revisión acerca de los instrumentos disponibles utilizados en otros países. Una de las características de estos instrumentos es que se encuentran sesgados hacia algunas áreas específicas de la creatividad artística -música, literatura, artedejando de lado otras donde la expresión de la creatividad es sumamente relevante-empresas y negocios, arquitectura-.

En este trabajo se diseñaron diferentes indicadores de comportamiento creativo referidos a las áreas de literatura; música; expresión corporal (danzas y teatro); diseño y artesanías; artes plásticas; empresas y negocios; arquitectura y construcción, ciencia y tecnología; y creatividad cotidiana. Los indicadores de comportamiento creativo diseñados apuntan a evaluar la creatividad en dominios particulares e independientes entre sí. Los comportamientos creativos en un área disciplinar no implican necesariamente la presencia de comportamientos o logros creativos en otras áreas. Sin embargo, las personas pueden mostrar habilidades $y$ comportamientos creativos en más de un área disciplinar.

Por último, los reactivos diseñados apuntan a evaluar la creatividad diaria. Como es sabido, obtener un premio en un área implica dedicación y formación en la misma. Por tal motivo se decidió incluir en la ECC reactivos que no sólo apuntaran a evaluar los logros obtenidos (e.g. "Ha publicado una pieza literaria -poema, cuento, etc.-") sino también reactivos más sutiles y que implicaran un menor grado de compromiso con la actividad aunque no su ausencia (e.g. "Ha participado en un taller o curso de escritura"). Por último, dado que el reconocimiento social es un elemento importante a la hora de hablar de creatividad -a pesar de no hablar de la creatividad sobresaliente- también se incluyeron reactivos que reflejaran esta particularidad (e.g. "Ha recibido un premio por algún logro en literatura").

\section{MATERIALES Y MÉTODOS}

\subsection{Participantes}

Para conformar la muestra se utilizó un muestreo no probabilístico-intencional. Participaron 258 sujetos, de los cuales un $67.4 \%(n=174)$ son de sexo femenino y 
un $32.6 \%$ ( $n=84)$, de sexo masculino. El rango de edad de los sujetos es de 18 a 60 años, presentando una media de 26.43 (DE $=10.73$ ). Para facilitar análisis posteriores, los participantes fueron clasificados en dos grupos: de 18 a 30 años, adultos jóvenes ( $n$ $=198) \mathrm{y}$ de 31 a 60 , adultos medios $(n=55)$.

En relación al nivel educativo de los participantes se observa que un $72.5 \%(n=187)$ completó sus estudios secundarios y un $27.5 \%$ $(n=71)$ finalizó los estudios terciarios o universitarios. También se indagó el área de estudios de los encuestados a fin de evaluar su posible influencia en los resultados. Un 56.2\% ( $n=$ 145) pertenece al área de ciencias sociales o humanas, un $5.8 \%$ a ciencias económicas ( $n$ $=15)$, un $5.4 \%$ a derecho $(n=14)$, un $4.7 \%$ a ciencias médicas $(n=12)$, un $4.3 \%$ a ciencias de la educación $(n=11)$, un $3.1 \%$ a ingeniería $(n=8)$, un $2.3 \%$ a arquitectura y diseño $(n=$ $6)$, un $0.4 \%$ a ciencias biológicas $(n=1)$, un $1.2 \%$ a ciencias exactas $(n=3)$, un $10.1 \%$ a otras (entre las que se incluyen ciencias de la comunicación, turismo, traductorado, analistas en sistemas, principalmente) $(n=26)$ y un $1.6 \%$ $(n=4)$ no contestan la pregunta.

Por último, en referencia al lugar de residencia de los encuestados, un $53.9 \%(n=139)$ pertenece a la Ciudad Autónoma de Buenos Aires y un $46.1 \%(n=119)$ reside en el Gran Buenos Aires.

\subsection{Instrumentos}

Escala de Comportamiento Creativo (ECC) diseñada y administrada con la finalidad de evaluar el comportamiento creativo en diferentes dominios, en población joven y adulta.

Escala de Personalidad Creativa (Creativity Personality Scale; Gough, 1979) (adaptación argentina Aranguren \& Irrazabal, 2010). La EPC consta de 30 ítems que evalúan el grado de acuerdo del sujeto con distintos adjetivos presentados, que se relacionan con la personalidad creativa. Siguiendo el estudio realizado por Dollinger, Dollinger y Centeno (2005), se utilizó una escala tipo likert de 5 puntos para cada ítem, cuyas opciones de respuestas son totalmente de acuerdo, bastante de acuerdo, de acuerdo, difícilmente de acuerdo y para nada de acuerdo. La escala está compuesta de 18 reactivos positivos y 12 reactivos negativos. En virtud de estudios realizados, se observan adecuadas propiedades psicométricas. En el presente estudio el alpha de Cronbach fue de .550. En el trabajo realizado por Dollinger et al. se reporta un alpha de Cronbach de .650 .

Escala de Autoeficacia General (Generalized Self-Efficacy Scale; Jerusalem \& Schwarzer, 1992), en su adaptación argentina (Brenlla, Aranguren, Rossaro \& Vázquez, 2010), compuesta por 10 ítems, evaluados cada uno con una escala tipo likert con cuatro opciones de respuesta -nunca, pocas veces, a veces o siempre - a las cuales se les otorga una puntuación de 1, 2, 3 y 4 respectivamente. Las puntuaciones varían entre los 10 y 40 puntos. Los valores más altos indican una mayor percepción de autoeficacia. En el presente estudio el alpha de Cronbach fue de .782. En estudios anteriores, tanto nacionales como internacionales, se encuentran índices de confiabilidad similares al reportado (Brenlla et al., 2009; Schwarzer, 1997).

Escala de Autoeficacia creativa (Creativity Self-Efficacy Scale; Yi, Scheithauer, Lin \& Schwarzer, 2008) en su adaptación argentina (Aranguren, Oviedo \& Irrazabal, 2011). Dicha escala consta de 5 ítems, evaluados cada uno con una escala tipo likert con 4 opciones de respuesta, a las cuales se le otorga una puntuación de 1, 2, 3 y 4 respectivamente. Las puntuaciones varían entre los 5 y los 20 puntos, indicando los valores más altos una mayor percepción de autoeficacia. En el presente estudio el alpha de Cronbach fue de .675, mientras que en el estudio original de la escala se reporta un alpha de .83 (Yi et al., 2008).

\subsection{Procedimiento y análisis de datos}

En primer lugar, se realizó una investigación bibliográfica acerca de los diferentes instrumentos disponibles para la evaluación de la creatividad. Considerando esta revisión y en función del objetivo de trabajo propuesto - diseñar un instrumento capaz de evaluar la creatividad en distintos dominios- se formularon nueve dimensiones para la agrupación de los indicadores: literatura; 
música; expresión corporal (danza y teatro); diseño y artesanías; artes plásticas; empresas y negocios; arquitectura y construcción, ciencia y tecnología; y creatividad cotidiana. Estas subdimensiones no obedecen a una clasificación teórica específica, sino que fueron generadas con el propósito de facilitar la organización y el análisis del material procedente de la literatura y de las entrevistas con expertos.

En relación a la información recolectada en base a la literatura revisada, se debe señalar que muchos de los indicadores propuestos en la versión piloto de la escala fueron incluidos atendiendo especialmente al Inventario de Comportamiento Creativo de Hocevar $(1979,1980)$ y al Cuestionario de Logros Creativos (Carson et al., 2005). En relación a las entrevistas con expertos, dichas entrevistas fueron realizadas con la finalidad de obtener información acerca de la creatividad, específicamente en las áreas de ciencia y tecnología, empresas y negocios y, arquitectura y construcción. Por último, para la selección de los reactivos que iban a ser incluidos en la versión piloto de la escala se tuvieron en cuenta los criterios de representatividad y relevancia propuestos por Martínez Arias (1996).

Una vez construidos los ítems y elaborado el formato de la escala (auto-administrable), se procedió a redactar las instrucciones de la misma. La consigna dada fue la siguiente:

A continuación se presentan una lista de actividades y logros que pueden ser considerados como creativos.

Por favor, indique la respuesta que mejor describa la frecuencia en la que se da ha dado este tipo de comportamiento a lo largo de su vida. Asegúrese de contestar todas las preguntas.

En segundo lugar, se realizó una administración piloto a 50 participantes entre 17 y 60 años. De los 82 reactivos iniciales fueron eliminados dos, en virtud de los resultados obtenidos en esta etapa. Algunos ítems fueron modificados para mejorar su comprensión, teniendo en cuenta las sugerencias de los sujetos. La consigna no ofreció dificultades; sin embargo, el formato de respuesta fue modificado teniendo en cuenta las observaciones de los participantes. Así, el formato original de respuesta, solicitaba al sujeto señalar en una escala de tipo likert de 5 puntos, la cantidad de veces que había realizado determinado comportamiento: Nunca, Una vez, Dos veces, Tres veces, Cuatro veces o más. Se reemplazó la leyenda o etiqueta de esta escala, por una más sencilla y general, debido a que muchos de los entrevistados alegaban no recordar con exactitud cuántas veces habían realizado tal o cual actividad. El formato de escala para la versión final incluida en la toma fue: Nunca, Alguna Vez, Pocas veces, Varias veces $y$, Muchas veces.

\section{RESULTADOS}

\subsection{Análisis factorial}

Se exploró la estructura factorial de la escala con el método de análisis de componentes principales. Con el objetivo de simplificar la lectura de la matriz factorial se suprimieron los valores absolutos menores que .35. El valor del test de adecuación muestral Kaiser Meyer Olkin fue de .784 y la significación del test de esferidad de Bartlett fue de $.000\left(\chi^{2}(3160)=\right.$ 10051.98). Se eliminaron aquellos ítems con carga inferior a 0.35 y los ítems complejos (aquellos que pesaban alto en más de un factor). A partir de la observación del gráfico de sedimentación (Pendiente de Catell) en los posteriores análisis factoriales exploratorios se extrajeron 4 factores. Se realizaron varios análisis factoriales exploratorios a través del método de componentes principales con rotación ortogonal (Varimax) para poder obtener una estructura factorial consistente. Finalmente, se obtuvieron 37 reactivos agrupados en 4 factores unipolares que explicaban un $47.66 \%$ de la varianza total. El valor del test de adecuación muestral Kaiser Meyer Olkin fue de .816 y la significación del test de esfericidad de Bartlett fue de .000 ( $\chi 2$ $(666)=4149.1)$. Los 4 factores obtenidos son los siguientes: (a) Diseño y artes con 13 ítems (F1); (b) Literatura y música con 9 ítems (F2); (c) Expresión corporal con 7 ítems (F3) y (d) Empresas y negocios con 8 ítems. En la Tabla 2 (página siguiente) se observa la distribución factorial de los reactivos de la escala. 
Tabla 2.

Resultados del análisis factorial exploratorio de la Escala de Comportamiento Creativo.

\begin{tabular}{|c|c|c|c|c|}
\hline & F1 & $\mathbf{F 2}$ & F3 & F4 \\
\hline Ha pintado un cuadro original & .653 & & & \\
\hline $\begin{array}{l}\text { Ha realizado artesanias en plástico, acrilico, vitral, resina o materiales } \\
\text { similares. }\end{array}$ & 686 & & & \\
\hline Ha decorado su propia casa. & .473 & & & \\
\hline $\begin{array}{l}\text { Ha realizado una escultura (excluyendo trabajos realizados en la escuela o } \\
\text { en la universidad). }\end{array}$ & .593 & & & \\
\hline Ha dibujado caricaturas o historietas. & .536 & & & \\
\hline Ha disefiado y/o confeccionado vestimenta & .442 & & & \\
\hline Ha diseñado y mantenido un jardín & .366 & & & \\
\hline Ha encontrado usos novedosos para objetos de uso cotidiano & .411 & & & \\
\hline Ha llevado un cuaderno de bocetos & 676 & & & \\
\hline Ha fabricado artesanias en cerámica & .597 & & & \\
\hline Ha realizado una exposición de arte o artesanias & .588 & & & \\
\hline Ha confeccionado un arreglo floral original & .442 & & & \\
\hline $\begin{array}{l}\text { Ha participado en talleres y/o clases de dibujo (excluyendo actividades } \\
\text { realizadas dentro del ámbito escolar). }\end{array}$ & .669 & & & \\
\hline Ha dado un recital & & .764 & & \\
\hline $\begin{array}{l}\text { Ha escrito poemas (excluyendo trabajos realizados en la escuela o en la } \\
\text { universidad). }\end{array}$ & & .517 & & \\
\hline Ha participado en un concurso de canto (como solista o en un coro) & & .494 & & \\
\hline Ha escrito la letra de una canción & & .718 & & \\
\hline Ha formado parte de una banda o conjunto musical & & .837 & & \\
\hline $\begin{array}{l}\text { Ha escrito un cuento (excluyendo trabajos realizados en la escuela o en la } \\
\text { universidad). }\end{array}$ & & .395 & & \\
\hline Ha participado en un concurso como músico & & .719 & & \\
\hline Ha compuesto música para tocar con uno o más instrumentos & & .783 & & \\
\hline Ha recibido premio por logros musicales & & .680 & & \\
\hline Ha recibido un premio en danzas & & & .734 & \\
\hline Ha realizado la coreografia de un baile & & & .762 & \\
\hline Ha desempeñado un papel en una obra de teatro & & & .534 & \\
\hline Ha entrado a un concurso o presentación de ballet o danzas modernas & & & .809 & \\
\hline Ha participado en exposiciones de comedia musical, danza o canto & & & .787 & \\
\hline Ha asistido a clases de danza. & & & .836 & \\
\hline Ha participado en un taller de teatro $u$ actuación & & & .631 & \\
\hline $\begin{array}{l}\text { Ha creado una empresa u organización que brinde un servicio nuevo (ej. } \\
\text { delivery, ONG). }\end{array}$ & & & & .803 \\
\hline Ha diseñado un producto y logrado su inserción en el mercado & & & & .750 \\
\hline Ha dirigido o administrado una empresa & & & & .737 \\
\hline Ha innovado en determinados productos o servicios ya existentes. & & & & .664 \\
\hline $\begin{array}{l}\text { Ha introducido alguna innovación en cualquiera de los procesos o fases } \\
\text { que conllevan a la realización de un negocio. }\end{array}$ & & & & .708 \\
\hline Ha creado y patentado una nueva marca & & & & .729 \\
\hline Ha puesto en marcha un microemprendimiento con buenos resultados & & & & .818 \\
\hline $\begin{array}{l}\text { Ha invertido en un producto, emprendimiento o proyecto con buenos } \\
\text { resultados. }\end{array}$ & & & & .702 \\
\hline Porcentaje de la varianza explicado & 11.87 & 12.12 & 11.35 & 12.32 \\
\hline
\end{tabular}

\subsection{Análisis del poder discriminativo de los ítems}

Para analizar la capacidad discriminativa de los ítems, se calcularon las medias y varianzas de cada uno de los sujetos comprendidos en el cuartil inferior y superior y se aplicó la prueba $t$ de Student de diferencia de medias. Se encontró un alto poder discriminativo para los 37 ítems de la Escala de Comportamiento Creativo.

\subsection{Análisis de la consistencia interna de la escala}

Una vez realizado el análisis factorial y el análisis del poder discriminativo de los ítems, se procedió a calcular el alpha de Cronbach para la escala y sus respectivas subescalas. La versión final de la escala, obtenida luego del análisis factorial, presentó un adecuado nivel de alpha de Cronbach $(\alpha=.844)$, siendo el alpha para 
la dimensión de Artes y diseño de .822 , para la dimensión de Literatura y música de .837 , para Expresión corporal de .867 y, por último, para Empresas y negocios de .879. Estos resultados indican que tanto la ECC como sus respectivas subescalas presentan índices de consistencia interna satisfactorios. En relación a este procedimiento, se ha de señalar que todos los ítems fueron conservados dado que la eliminación de ningún reactivo aumentaba el alpha de las subescalas y de la escala total.

\subsection{Evidencias de validez convergente}

Para poder obtener evidencias de validez convergente, es necesario disponer de instrumentos que evalúen un mismo rasgo o constructo, sea en forma completa o parcial, de tal forma que las evidencias de convergencia estarán dadas por las correlaciones relativamente altas entre sus respectivas puntuaciones (Tornimbeni, Pérez \& Olaz, 2008). En este sentido, es relevante mencionar que no se cuenta en nuestro medio con escalas o cuestionarios que evalúen de manera precisa, actividades o comportamientos creativos, de ahí la necesidad de diseñar uno. Por lo que, para realizar el análisis de la validez convergente se incluyeron en la administración instrumentos cercanos, teóricamente, al constructo a evaluar, pero no exactos en su delimitación y especificidad. Los instrumentos incluidos para este fin fueron, la Escala de Personalidad Creativa (EPC) (Gough, 1979; adaptación Aranguren \& Irrazabal, 2010), la Escala de Autoeficacia Creativa (EAC) (Yi, Scheithauer, Lin \& Schwarzer, 2008; adaptación Aranguren, Oviedo \& Irrazabal, 2011) y la Escala de Autoeficacia General (EAG) (Jerusalem \& Schwarzer, 1992; adaptación Brenlla, Aranguren, Vazquéz \& Rossaro, 2010).

En relación a la inclusión de la EPC, se ha de señalar que diferentes estudios han evidenciado que determinados rasgos de personalidad se encuentran asociados a la creatividad (Gough, 1979), permitiendo de esta manera, estimar el potencial creativo de las personas a través de la evaluación de la presencia o ausencia de determinados rasgos característicos. Por su parte, la autoeficacia creativa consiste en la creencia acerca de la capacidad de uno mismo para generar productos creativos (Tierney \& Farmer, 2002). De este modo se entiende que los productos o resultados creativos se ven influenciados por los juicios que las personas tienen acerca de sus capacidades para generar resultados útiles y originales. A partir de esto, se induce que tanto la autoeficacia general, como la autoeficacia creativa, específicamente, pueden ser predictores adecuados del comportamiento creativo. De ahí su inclusión en el presente estudio. En la tabla 3 se muestran los resultados de los análisis realizados.

Tabla 3.

Correlaciones entre la ECC y otras medidas seleccionadas.

\begin{tabular}{|lrrr|}
\hline & EPC & EAC & EAG \\
\hline ECC & $.150^{\star}$ & $.224^{\star \star}$ & .123 \\
$\boldsymbol{n}$ & 219 & 236 & 234 \\
Artes y Diseño. & .047 & $.170^{\star *}$ & .079 \\
$\boldsymbol{N}$ & 228 & 247 & 245 \\
Literatura y Música & $.209^{\star *}$ & $.220^{\star *}$ & .093 \\
$\boldsymbol{N}$ & 231 & 252 & 251 \\
Expresión Corporal & .120 & -.017 & -.046 \\
$\boldsymbol{N}$ & 228 & 248 & 247 \\
Empresas y Negocios & -.040 & $.174^{\star \star}$ & $.229^{\star \star}$ \\
$\boldsymbol{n}$ & 230 & 250 & 249 \\
$p<.01^{\star \star} ; p<.05^{\star}$ & & & \\
\hline
\end{tabular}

\subsection{Evidencias de validez de criterio por grupos contrastados}

Las evidencias de validez de criterio están referidas al grado en que las puntuaciones totales de un instrumento correlacionan con las de un criterio externo, siendo evaluadas en un mismo momento (Martínez Arias, 1996). De acuerdo con esto, en el cuestionario de datos sociodemográficos de este trabajo, se incluyó la siguiente pregunta: "Realiza o ha realizado alguna actividad artística", donde el participante debía responder por sí o por no. A su vez, a modo ilustrativo, también se le presentaba una lista de actividades que eran consideradas artísticas entre las que se nombraban: pintura, escultura, escritura, danza, teatro, fotografía, dibujo, canto, instrumentos musicales, gimnasia artística y cinematografía.

Con la finalidad de tener otras evidencias de validez y, luego de haber realizado el análisis factorial exploratorio de la escala -la cual incluye en su mayoría indicadores referidos a diferentes actividades artísticas- se decidió realizar un análisis de grupos contrastados empleando un prueba $t$ de diferencia de medias para muestras independientes. Así se compararon las puntuaciones totales promedios para la ECC del grupo que realizaba o había realizado alguna actividad 
artística y las puntuaciones promedios para ECC y las distintas subescalas del grupo que nunca había realizado una actividad artística. Tal como puede observarse en la tabla 4, tanto la ECC como las subescalas de Artes y diseño, Literatura y música y Expresión corporal, diferencian los grupos contrastados. Por otro lado, también se puede observar que las puntuaciones promedios de los sujetos que realizan o han realizado una actividad artística, tanto para la ECC como para las subescalas mencionadas, son ampliamente mayores que la de los sujetos que no han realizado una actividad artística. No sucede lo mismo en la subescala de Empresas y negocios, encontrándose esto en concordancia con lo planteado. Debido a que el criterio externo escogido refiere a las actividades artísticas realizadas, dicho criterio no es influyente en lo que refiere a actividades creativas empresariales o de negocios, por lo que resulta esperable no encontrar diferencias en las puntuaciones promedios de esta subescala en los grupos contrastados.

Tabla 4.

Comparación de los valores medios entre los participantes que realizan o han realizado una actividad artística y aquellos que no.

\begin{tabular}{|c|c|c|c|c|c|c|c|c|}
\hline & $\begin{array}{l}\text { Actividad } \\
\text { artistica. }\end{array}$ & $n$ & M & $D E$ & $t$ & $g l$ & $p$ & $d$ \\
\hline \multirow{2}{*}{ ECC } & $\mathrm{Si}$ & 187 & 70.37 & 15.60 & \multirow{2}{*}{7.169} & \multirow{2}{*}{233} & \multirow{2}{*}{.000} & \multirow{2}{*}{1.16} \\
\hline & No & 48 & 53.10 & 11.60 & & & & \\
\hline \multirow{2}{*}{$\begin{array}{l}\text { Artes y } \\
\text { Diseño }\end{array}$} & $\mathrm{Si}$ & 194 & 26.41 & 8.65 & \multirow{2}{*}{4.209} & \multirow{2}{*}{244} & \multirow{2}{*}{.000} & \multirow{2}{*}{5} \\
\hline & No & 52 & 20.94 & 6.91 & & & & \\
\hline \multirow{2}{*}{$\begin{array}{l}\text { Literatura y } \\
\text { Música }\end{array}$} & $\mathrm{Si}$ & 198 & 16.68 & 7.08 & \multirow{2}{*}{4.666} & \multirow{2}{*}{250} & \multirow{2}{*}{.000} & \multirow{2}{*}{2} \\
\hline & No & 54 & 12.02 & 3.63 & & & & \\
\hline \multirow{2}{*}{$\begin{array}{l}\text { Expresión } \\
\text { Corporal }\end{array}$} & $\mathrm{Si}$ & 194 & 16.20 & 7.15 & \multirow{2}{*}{5.556} & \multirow{2}{*}{246} & \multirow{2}{*}{.000} & \multirow{2}{*}{.86} \\
\hline & No & 54 & 10.50 & 4.45 & & & & \\
\hline \multirow{2}{*}{$\begin{array}{l}\text { Empresas y } \\
\text { Negocios }\end{array}$} & $\mathrm{Si}$ & 197 & 10.71 & 5.06 & \multirow{2}{*}{.496} & \multirow{2}{*}{248} & \multirow{2}{*}{.620} & \multirow{2}{*}{.07} \\
\hline & No & 53 & 10.34 & 3.81 & & & & \\
\hline
\end{tabular}

\subsection{Influencia de sexo, edad y nivel educativo}

Se encontraron diferencias significativas por sexo en las puntuaciones totales de la ECC ( $t$ $(237)=3.45, p=.001, d=.48)$ y en las subescalas de Artes y diseño $(t(248)=3.13, p=.002$, $d=.43)$, Expresión corporal $(t(250)=9.67, p=$ $.000, d=1.31)$ y Empresas y negocios $(t(252)=$ $-3.37, p=.001, d=-.45)$. No se encontraron dichas diferencias en la subescala de Literatura y música $(t(254)=-2.21, p=.028, d=-.29)$ En la tabla 5 se muestran las medias y desvíos estándar par a las puntuaciones totales de la ECC y cada una de sus subescalas según sexo.

En relación a la edad se encontraron diferencias significativas en las puntuaciones totales de la ECC $(t(233)=2.82 ; p=.005, d=.45)$ y las subescalas de Literatura y música $(t(249)=3.98$, $p=.000, d=.60)$, Expresión corporal $(t(246)=$ $5.51, p=.000, d=.84)$ y Empresas y negocios $(t(247)=-5.17, p=.000, d=-.79)$. No se encontraron diferencias significativas en la subescala de de Artes y diseño $(t(244)=.874, p=.383, d$ $=.13$ ). Por último, en relación a al nivel educativo se encontraron diferencias significativas en las subescalas de Expresión corporal $(t(250)=$ $2.88, p=.004, d=40)$ y Empresas y negocios $(t$ $(252)=-3.06, p=.002, d=-.43)$. No se hallaron diferencias significativas en las puntuaciones totales de la ECC $(t(237)=1.13, p=.256, d=$ .16) y en las subescalas de Artes y diseño $(t$ (248) $=-.091, p=.928, d=-.13)$, y de Literatura y música $(t(254)=1.85, p=.064, d=.25)$. En las tablas 6 y 7 muestran las medias y desvíos estándar par a las puntuaciones totales de la ECC y cada una de sus subescalas según rangos de edad y nivel educativo respectivamente.

Tabla 5.

Medias y Desvíos estándar para las puntuaciones totales de la EPC y cada una de las subescalas según sexo.

\begin{tabular}{|c|c|c|c|c|}
\hline & & $n$ & $M$ & $D E$ \\
\hline \multirow{2}{*}{ ECC } & Femenino & 162 & 69.24 & 16.86 \\
\hline & Masculino & 77 & 61.59 & 13.89 \\
\hline \multirow{2}{*}{$\begin{array}{l}\text { Artes y } \\
\text { Diseño }\end{array}$} & Femenino & 170 & 26.41 & 9.02 \\
\hline & Masculino & 80 & 22.84 & 6.93 \\
\hline \multirow{2}{*}{$\begin{array}{l}\text { Literatura } \\
\text { y Música }\end{array}$} & Femenino & 173 & 15.05 & 5.86 \\
\hline & Masculino & 83 & 17.02 & 8.18 \\
\hline \multirow{2}{*}{$\begin{array}{l}\text { Expresión } \\
\text { corporal }\end{array}$} & Femenino & 170 & 17.42 & 7.03 \\
\hline & Masculino & 82 & 9.60 & 2.85 \\
\hline Empresas & Femenino & 171 & 9.94 & 3.75 \\
\hline Negocios & Masculino & 83 & 12.07 & 6.25 \\
\hline
\end{tabular}

Tabla 6.

Medias y Desvíos estándar para las puntuaciones totales de la EPC y cada una de las subescalas según rangos de edad.

\begin{tabular}{|c|c|c|c|c|}
\hline & & $n$ & $M$ & $D E$ \\
\hline \multirow{2}{*}{ ECC } & Adultos jóvenes & 186 & 68.45 & 16.79 \\
\hline & Adultos medios & 49 & 61.16 & 13.06 \\
\hline \multirow{2}{*}{$\begin{array}{l}\text { Artes y } \\
\text { Diseño }\end{array}$} & Adultos jóvenes & 196 & 25.54 & 8.79 \\
\hline & Adultos medios & 50 & 24.36 & 7.80 \\
\hline \multirow{2}{*}{$\begin{array}{l}\text { Literatura } \\
\text { y Música }\end{array}$} & Adultos jóvenes & 196 & 16.55 & 7.03 \\
\hline & Adultos medios & 55 & 12.55 & 4.53 \\
\hline \multirow{2}{*}{$\begin{array}{l}\text { Expresión } \\
\text { corporal }\end{array}$} & Adultos jóvenes & 193 & 16.17 & 7.15 \\
\hline & Adultos medios & 55 & 10.55 & 4.57 \\
\hline Empresas & Adultos jóvenes & 195 & 9.88 & 3.74 \\
\hline Negocios & Adultos medios & 54 & 13.56 & 6.93 \\
\hline
\end{tabular}


Tabla 7.

Medias y Desvíos estándar para las puntuaciones totales de la EPC y cada una de las subescalas según nivel educativo.

\begin{tabular}{|c|c|c|c|c|}
\hline & & $n$ & $M$ & $D E$ \\
\hline \multirow{2}{*}{ ECC } & Secundario Completo & 172 & 67.52 & 15.94 \\
\hline & Terc./Universitario Completo & 67 & 64.85 & 17.26 \\
\hline \multirow{2}{*}{$\begin{array}{l}\text { Artes y } \\
\text { Diseño }\end{array}$} & Secundario Completo & 181 & 25.24 & 8.33 \\
\hline & Terc./Universitario Completo & 69 & 25.35 & 9.19 \\
\hline \multirow{2}{*}{$\begin{array}{l}\text { Literatura } \\
\text { y Música }\end{array}$} & Secundario Completo & 186 & 16.17 & 6.97 \\
\hline & Terc./Universitario Completo & 70 & 14.41 & 6.00 \\
\hline \multirow{2}{*}{$\begin{array}{l}\text { Expresión } \\
\text { corporal }\end{array}$} & Secundario Completo & 181 & 15.66 & 7.46 \\
\hline & Terc./Universitario Completo & 71 & 12.86 & 5.31 \\
\hline Empresas & Secundario Completo & 184 & 10.08 & 4.15 \\
\hline Negocios & Terc./Universitario Completo & 70 & 12.11 & 6.00 \\
\hline
\end{tabular}

\section{DISCUSIÓN Y CONCLUSIONES}

El punto de partida de este trabajo fue diseñar un instrumento capaz de evaluar la creatividad en diferentes dominios. Dicho objetivo fue planteado a raíz de la escasez de instrumentos disponibles en nuestro medio para la medición de este atributo. Teniendo en cuenta que el estudio de la creatividad a nivel internacional ha ido creciendo a partir de la década del ' 50 y ha cobrado especial relevancia en los últimos años en el ámbito del descubrimiento, de la innovación y de la salud, se considera necesario contar con pruebas adecuadas para su medición.

En un primer momento, basándose en una revisión de los instrumentos utilizados a nivel internacional y en función de las entrevistas realizadas con expertos, se diseñaron 82 reactivos referidos a nueve dominios en que puede tener lugar la creatividad: (a) literatura; (b) música; (c) expresión corporal (danzas y teatro); (d) diseño y artesanías; (e) artes plásticas; (f) empresas y negocios; (g) arquitecturas y construcción; (h) ciencia y tecnología y; (i) creatividad cotidiana. En un segundo momento, se administró la versión preeliminar de la escala a 50 participantes para poder examinar si los ítems, la consigna y la leyenda de la escala likert eran comprendidos adecuadamente. En función de los resultados obtenidos en esta etapa, se eliminaron dos ítems y algunos ítems fueron modificados para poder mejorar su comprensión.

En un tercer momento, se administró la escala a un total de 258 participantes y se prosiguió a efectuar el análisis de las propiedades psicométricas de la escala. En referencia a las evidencias de validez de constructo, de los nueve dominios planteados inicialmente, seis fueron incluidos en la versión final de la escala -artes plásticas, artesanías y diseño, literatura, música, expresión corporal y empresas y negocios-. En tanto que los tres dominios restantes -ciencia y tecnología, arquitectura y construcción y creatividad cotidiana-, debieron ser dejados de lado una vez realizado el análisis factorial exploratorio de la escala. La estructura factorial obtenida agrupa los indicadores principales de las seis áreas mencionadas en cuatro factores. Los 37 reactivos incluidos presentan cargas factoriales altas en cada una de las dimensiones. Dado que los resultados del análisis factorial son sensibles a los datos ingresados, futuras investigaciones deberán ser realizadas con muestras obtenidas aleatoriamente o contemplando la inclusión de grupos muestrales específicos de cada una de las áreas a fin de poder incluir un mayor número de dominios y reactivos.

En lo que respecta a las evidencias de fiabilidad de la versión final de la escala se observa un alto poder discriminativo de los ítems y un coeficiente de alpha satisfactorio tanto para la escala en su totalidad como para cada una de sus subescalas. En relación a las evidencias de validez convergente, se ha de señalar que muchas de las correlaciones encontradas fueron bajas aunque significativas estadísticamente. Esto podría deberse a la falta de instrumentos -adaptados en nuestro medio- destinados a medir el constructo aquí señalado. Teniendo en cuenta que los constructos evaluados por la ECC, EPC, EAG y EAC no son equivalentes, sino que son constructos que se relacionan teóricamente -comportamiento creativo, personalidad creativa, autoeficacia general y autoeficacia creativa-, los resultados obtenidos se consideran adecuados. Tanto los rasgos de personalidad como la autoeficacia son dos constructos que contribuyen a explicar el comportamiento creativo, enfatizando cada uno diferentes aspectos que pueden influir en el mismo. Sin embargo, hay muchas otros aspectos que condicionan la creatividad de la persona como pueden ser las destrezas relevantes para el campo y la motivación por la tarea (Amabile, 1983) y que deberían tenerse en consideración al valorarse estos resultados.

Al examinar las evidencias de validez de criterio por grupos contrastados se pueden observar 
diferencias estadísticamenentre significativas en las puntuaciones promedios para el total de la ECC y en las subescalas de Artes y diseño, Literatura y música y Expresión corporal entre los participantes que habían desarrollado alguna actividad artística versus aquellos participantes que no habían realizado ninguna actividad de esta naturaleza. Esta diferencia no se encuentra en las puntuaciones promedio de la subescala de Empresas y negocios. Dado que la ECC se encuentra destinada a la evaluación de la creatividad diaria y que un indicador de dicha creatividad es el compromiso en la realización de distintas actividades artísticas, se considera que estos resultados aportan otras evidencias acerca de la validez de la prueba.

En relación a las influencias de sexo se encontraron diferencias significativas en las puntuaciones totales de la ECC y en las subescalas de Artes y diseño, Expresión corporal y Empresas y negocios. En relación a la edad se encontraron diferencias significativas en la puntuaciones totales de la ECC, y en las subescalas de de Literatura y música, Expresión corporal y Empresas y negocios. Por último, en función del nivel educativo se hallaron diferencias en las subescalas de Expresión corporal y Empresas y negocios. Sin embargo se ha de señalar que una de las limitaciones del estudio fue no haber logrado un número equivalente de sujetos en relación al sexo, nivel educativo y rango de edades en la composición de la muestra. Esto puede haber influido en los resultados mencionados.

Por último, sobre la base de resultados expuestos, se puede decir que la ECC es un instrumento adecuado para evaluar la creatividad en los contextos de artes y diseño, literatura y música, expresión corporal y empresas y negocios. Estos resultados preliminares indican una buena consistencia interna en cada una de sus subescalas y una clara estructura factorial. Futuras investigaciones deberán ser realizadas a fin de aportar datos acerca de aquellos dominios de la creatividad que no fueron adecuadamente representados en la muestra de este estudio.

La escala puede ser aplicada en ámbitos educativos y de investigación. Es una escala breve, de fácil administración y puntuación que permite conocer el rendimiento y las actividades creativas de los alumnos y/o participantes de una manera sencilla y confiable. Este trabajo es un aporte a un área de investigación prometedora y necesaria como lo es el estudio de la creatividad.

\section{REFERENCIAS}

Amabile, T. (1983). The social psychology of creativity. New York: Springer-Verlag.

Aranguren, M. \& Irrazabal, N. (2010). Adaptación de la Escala de Personalidad Creativa de Gough. Manuscrito no publicado.

Aranguren, M., Oviedo, A. B. \& Irrazabal, N. (2011, en prensa). Estudio exploratorio de las propiedades psicométricas de la Escala de Autoeficacia Creativa. Revista de Psicología de la Pontificia Universidad Católica Argentina.

Armesto, M. C. (2001). Creatividad y autoconcepto: Un estudio con estudiantes de Psicología. Revista Interamericana de Psicología, 35 (1), 79-95.

Baer, J. (1998). The case for domain specificity of creativity. Creativity Research Journal, 11 (2), 173-177.

Baer, J., \& Kaufman, J. C. (2005). Bridging generality and specificity: The amusement park theoretical (APT) model of creativity. Roeper Review: A Journal on Gifted Education 27 (3), 158-163.

Barron, F. (1955). The disposition toward originality. Journal of Abnormal and Social Psychology, 51, 478-485.

Beghetto, R. A., \& Kaufman, J. C. (2007). Toward a broader conception of creativity: A case for "mini-c" creativity. Psychology of Aesthetics, Creativity, and the Arts, 1 (2), 73-79.

Beghetto, R. A. \&, Plucker, J. A. (2007). Darwinian creativity in the classroom? Creativity Research Journal, 19 (4), 375-379.

Brenlla, M.E., Aranguren, M., Rossaro F.,\& Vazquéz, N. (2010). Adaptación argentina de la Escala de Autoeficacia General de Jerusalem y Schwarzer. Revista Interdisciplinaria, 27,77-94.

Carson, S., Peterson, J. B., \& Higgings, D. M. (2005). Reliability, validity and factor structure of Creative Achievement Questionnaire. Creativity Research Journal, 17 (1), 37-50.

Csikszentmihalyi, M. (1998). Creatividad. El fluir y la psicología deldescubrimientoyla invención. Buenos Aires: Paidós.

Dollinger, S. J., S. M. Clancy Dollinger and L. Centeno. 2005. Identity and creativity. Identity: An International Journal of Theory and Research, Vol. 5, 4, pp. 315-339

Donolo, D. S. \& Elisondo, R. C. (2007). Creatividad para todos. Consideraciones sobre un grupo particular. Anales de Psicología, 23 (1), 147-151.

Donolo, D. \& Rinaudo, M. C. (2008). Perspectivas y experiencias creativas para estudiantes universitarios. Cuadernos FHyCS-UNJu, 35, 91-113.

Garaigordobil, M. (2004). Juegos cooperativosy creativos para grupos de niños de 10 a 12 años. España: Pirámide.

Gough, H.G. (1979). A creativity personality scale for the adjective checklist. Journal of Personality and Social Psychology, 37, 1398-1405.

Hayes, J. R. (1981). The complete problem solver. Filadelfia: Franklin Institute Press. 
Hocevar, D. (1979). The development of the Creative Behavior Inventory. Paper presented at the annual meeting of the Rocky Mountain Psychological Association. (ERIC Document Reproduction Service No. ED 170 350).

Hocevar, D. (1980). Intelligence, divergent thinking and creativity. Intelligence, 4, 25-40.

Hocevar, D. (1981). Measurement of creativity: Review and critique. Journal of Personality Assessment, 45 (5), 450-464.

Hocevar, D. \& Bachelor, P. (1989). A taxonomy and critique of measurement used in the study of creativity. En J. A. Glover, R. R. Ronning, \& C. R. Reynolds (Eds.), Handbook of creativity (pp. 53-75). New York: Plenum.

Holland, J. L. \& Nichols, R. C. (1964). Prediction of academic and extracurricular achievement in college. Journal of Educational Psychology, 55, 55-65.

Jerusalem, M. \& Schwarzer, R. (1992). Self-efficacy as a resource factor in stress appraisal process. En $\mathrm{R}$. Schwarzer (Ed.), Self-efficacy: Thought control of action (pp. 195-211). Washington, D.C.: Hemisphere.

Krumm, G. L. (2004). Creatividad verbal y rendimiento académico en estudiantes universitarios. Revista Internacional de Estudios en Educación, 4(2), 85-108.

Krumm, G. L. \& Lemos, V. N. (2010). Análisis preliminar de la validez del Test de Pensamiento Creativo de Torrance verbal Forma B. Acta Psiquiátrica y Psicológica de América Latina, 56(3), 168-173.

Krumm, G., \& Lemmos, V. (2011). Estudio exploratorio de las propiedades psicométricas de la Escala de Personalidad Creadora (EPC) en su versión heteroevaluación. Revista Interamericana de Psicología, 45 (1), 21-28.

Ludwig, A. (1992). The Creative Achievement Scale. Creativity Research Journal, 5, 109-124.

MacKinnon, D. W. (1962). The nature and nurture of creative talent. American Psychologist, 17, 484-495.

Martinez Arias, R. (1996). Psicometría: Teoría de los Tests Psicológicos y Educativos. Madrid: Síntesis.

Maslow, A. H. (1968). Toward a psychology of being. New York: Van Nostrand

Plucker, J. A. (1998). Beware of simple conclusions: The case for content generality of creativity. Creativity Research Journal, 11 (2), 179-182.

Plucker, J. A., Beghetto, R. A., \& Dow, G. T. (2004). Why isn't creativity more important to educational psychologists? Potentials, pitfalls, and future directions in creativity research. Educational Psychologist, 39, 83-96.

Plucker, J. A. \& Renzulli, J. S. (1999). Psychometric approaches to the study of human creativity. En R. J. Sternberg. (Ed.), Handbook of Creativity. (pp. 35-61). New York: University Press.
Real Academia Española. (2001). Diccionario de la lengua española (22.a ed.). Consultado en http://www.rae. es/rae.html

Richards, R. (2007). Everyday creativity and the arts. World Futures, 63, 500-525.

Richards, R., Kinney, D. K., Benet, M. \& Merzel, A. P. C. (1988). Assessing everyday creativity: Characteristics of the Lifetime Creativity Scales and validation with tree large samples. Journal of Personality and Social Psychology, 54 (3), 476-485.

Rogers, C. R. (1970). Toward a theory of creativity. En P. E. Vernoon (Ed.), Creativity (pp. 137-151). New York: Penguin.

Romo, M. (1997). Psicología de la Creatividad. Barcelona: Paidos.

Runco, M. A. (2003). Commentary on personal and potentially ambiguous creativity: You can't understand the butterfly unless you (also) watch the caterpillar. Creativity Research Journal, 15 (2\&3), 137-141.

Schwarzer, R., \& Born, A. (1997). Optimistic self-beliefs: Assessment of general perceived self-efficacy in thirteen cultures. World Psychology, 3(1-2), 177-190.

Sternberg, R. \& Lubart, T. (1999). The concept of creativity: Prospects and paradigms. En R. J. Sternberg (Ed.), Handbook of Creativity (pp. 3-15). New York: Cambridge University Press.

Tierney, P. \& Farmer, S. M. (2002). Creative self-efficacy: Potential antecedents and relationship to creative performance. Academy of Management Journal, 45, 1137-1148.

Tornimbeni, S., Pérez, E. \& Olaz, F. (2008). Introducción a la Psicometría. Buenos Aires: Paidós.

Torrance, E. P. (1972). Creative young women in today's world. Exceptional Children, 38, 597-603.

Torrance, E. P. (1974). Torrance Test of Creative Thinking: Norms-technical manual. Bensenville: Scholastic Testing Service.

Ward, T. B., Smith, S. M. \&, Finke, R. A. (1999). Creative cognition. En R. J. Sternberg (Ed.). Handbook of creativity, (pp. 189-212). New York: Cambridge University Press.

Weisberg, R. (2006). Modes of expertise in creative thinking: Evidence from case studies. En K. A. Ericsson, N. Charness, P. Feltovich \& R. Hoffman (Eds.), The Cambridge handbook of expertise and expert performance (pp. 761-787). New York: Cambridge University Press.

Yi, X., Scheithauer, H., Lin, C., \& Schwarzer, R. (2008). The impact of culture on general self-efficacy, creativity self-efficacy and cultural efficacy. Tesis de doctorado no publicada. Universidad de Berlín.

Para citar este artículo:

Aranguren, M. \& Irrazabal, N. (2012). Diseño de una Escala para la evaluación del Comportamiento Creativo en diferentes dominios. Ciencias Psicológicas VI (1): 29-41. 